Review Article

\title{
The Efficacy of Brucea javanica Oil Emulsion Injection as Adjunctive Therapy for Advanced Non-Small-Cell Lung Cancer: A Meta-Analysis
}

\author{
Wei Xu, Xinchan Jiang, Zhengyuan Xu, Tong Ye, and Qionghua Shi \\ School of International Pharmaceutical Business, China Pharmaceutical University, Nanjing, Jiangsu 211198, China \\ Correspondence should be addressed to Xinchan Jiang; 15211040641@stu.cpu.edu.cn
}

Received 5 August 2016; Revised 9 November 2016; Accepted 10 November 2016

Academic Editor: Wenyi Kang

Copyright (@) 2016 Wei Xu et al. This is an open access article distributed under the Creative Commons Attribution License, which permits unrestricted use, distribution, and reproduction in any medium, provided the original work is properly cited.

\begin{abstract}
Purpose. To evaluate the efficacy of Brucea javanica oil emulsion injection (BJOEI) in patients with advanced non-small-cell lung cancer (NSCLC) during chemotherapy. Method. Electronic database of EMBASE and PubMed and the conference proceeding of ASCO, CNKI, CBMdisc, VIP, and Wanfang database were searched to select RCTs comparing BJOEI plus chemotherapy with chemotherapy alone in the treatment of advanced NSCLC, until June 1, 2016. Two reviewers independently performed the analysis according to the inclusion and exclusion criteria. Review Manager 5.3 and STATA 12.0 were employed for data analysis. Result. Twenty-one studies including 2234 cases were included. The pooled result indicated that there were significant differences in ORR $(\mathrm{RR}=1.25 ; 95 \%$ CI: $1.14-1.36 ; P<0.00001)$, improvement of QOL $(\mathrm{RR}=1.87 ; 95 \%$ CI: $1.63-2.15 ; P<0.00001)$, nausea and vomiting $(\mathrm{RR}=0.67 ; 95 \% \mathrm{CI}: 0.46-0.98 ; P=0.04)$, leukopenia $(\mathrm{RR}=0.63 ; 95 \% \mathrm{CI}: 0.52-0.75 ; P<0.00001)$, but there was no difference in thrombocytopenia $(\mathrm{RR}=0.78 ; 95 \% \mathrm{CI}: 0.49-1.23 ; P=0.29)$. Begg's funnel plot and Egger's test indicated that no publication bias was found. The sensitivity analysis suggested the stability of the pooled result. Conclusion. The addition of BJOEI can enhance efficacy, improve QOL, and decrease incidence of nausea and vomiting and leukopenia for advanced NSCLC patients. However, higher quality RCTs are needed to further confirm this finding.
\end{abstract}

\section{Introduction}

As a prevalent and highly malignant carcinoma, lung cancer is the leading cause and the second leading cause of cancer death among men and women worldwide, respectively [1]. While the incidence of lung cancer has declined in some regions like North America and Europe, it is still high in China with the incidence rate of 733.3 per 100,000 in 2015 [2]. Moreover, with an annual growth rate of $26.9 \%$, the expected number of cases of lung cancer will reach 100 million in 2025, indicating that China might have the largest population of lung carcinoma patients around the world [3].

Lung cancer can be divided into two types: one is smallcell lung cancer and the other is non-small-cell lung cancer. It is estimated that the latter, of which the most common types are squamous cell carcinoma, large-cell carcinoma, and adenocarcinoma, accounts for $80 \% \sim 85 \%$ of all global lung cancer cases [4]. The treatments of non-small-cell lung cancer
(NSCLC) give first place to surgery, but most of the patients cannot receive the appropriate resection as they have reached an advanced stage when being diagnosed [5]. Standard firstline treatment for advanced NSCLC involves a combination of two drugs, including a platinum compound and a nonplatinum compound, such as paclitaxel and docetaxel, having indeed achieved favorable outcome [6]. However, the substantial toxicity incurred by chemotherapy, including gastroenteric reaction, hematotoxicity, nausea, and vomiting, should not be overlook, which is negatively correlated with quality of life (QOL) for NSCLC patients [7], especially for the advanced ones. Thus, how to reduce the burden of toxicity and achieve higher quality of life is the top priority on the clinical research agenda [8].

Traditional Chinese Medicines (TCMs) have become increasingly popular in the treatment of cancer in China [9]. Brucea javanica oil emulsion injection (BJOEI) is one of TCMs products, which takes Brucea Jen petroleum ether 
extracts as raw material and purified soybean lecithin as emulsifier [10], and is employed as adjunctive therapy in the treatment of lung carcinoma, brain metastasis of lung carcinoma, and gastrointestinal tumorigenesis. A great number of published studies have proved that BJOEI can perform a synergetic antitumor effect by improving tumor response, boosting Karnofsky Performance Score (KPS), reducing the incidence of adverse events and stimulating the immunity during chemotherapy or radiotherapy [11]. The pooled result of a meta-analysis showed that the addition of BJOEI to chemotherapy produced favorable outcomes for patients with advanced gastric cancer, including improvement of objective response and QOL and reduction of side effects such as neutropenia, thrombocytopenia, nausea, and vomiting [12].

BJOEI has been applied in clinical practice for advanced NSCLC patients since long time ago, but no relevant metaanalysis was conducted. Thus, we perform this meta-analysis to investigate the clinical efficacy of BJOEI plus chemotherapy in the treatment of advanced NSCLC.

\section{Methods}

2.1. Literature Source and Search Strategy. We searched and extracted eligible studies about BJOEI treatment of NSCLC from databases of PubMed, EMBASE, the conference proceeding of American Society of Clinical Oncology (ASCO), Chinese Biological Medical disc (CBMdisc), Chinese National Knowledge Infrastructure (CNKI), Chinese Scientific Journals Full-text Database (VIP), and Wanfang database. The key words applied in the search were as followed: "lung cancer", "non-small-cell lung cancer", "NSCLC", "Brucea javanica oil emulsion", "BJOEI", "Yadanzi", and "chemotherapy". The retrieved studies were regarded as potential source and reviewed manually. Moreover, although the published year of these literatures were unlimited, only English and Chinese literatures were accepted.

2.2. Inclusion and Exclusion Criteria. The following criteria were used for the literature inclusion. (1) The study design was confined to randomized controlled trials (RCTs) comparing platinum-contained chemotherapy alone with platinumcontained chemotherapy plus BJOEI for the NSCLC. (2) Study subjects who (a) were patients with stage III or IV NSCLC diagnosed pathologically and (or) cytologically; (b) had KPS $\geq 60$ and (or) time of survival $\geq 3$ months; (c) had outcomes of objective response rate (ORR) determined by World Health Organization (WHO) criteria or Response Evaluation Criteria in Solid Tumors (RECST), improvement of QOL evaluated by KPS, and adverse reactions assessed by WHO Recommendations for Grading of Acute and Subacute Toxicity; (d) had no chemotherapy contraindication before treatment and no significant abnormalities in liver, kidney, and heart function. The major exclusion criteria were as follows: (a) non-RCTs studies; (b) animal experiments, review, and other irrelevant studies; (c) no detailed data about ORR, improvement of QOL, and adverse events or no indicators for them; (d) single-arm study.
2.3. Endpoint Indicator. The outcomes included clinical efficacy, quality of life, and adverse effects. According to WHO criteria and RECST, the tumor response included complete response (CR), partial response (PR), stable disease (SD), and progressive disease (PD). The ORR was defined as CR + PR. Toxicity was graded from 0 to IV in severity on the basis of the WHO Recommendations. This meta-analysis only investigated the incidence of Grade II or above nausea and vomiting, leukopenia, and thrombocytopenia.

2.4. Data Extraction and Quality Assessment. Two reviewers independently extracted the information of the included study including name of author(s), publication year, number of patients in BJOEI group and control group, age, sex, chemotherapy regimen, stage of cancer, initial KPS, method of outcome ascertainment, study outcome, and detail of BJOEI treatment. Disagreement and problems were resolved by discussing or consulting with another reviewer according to the Cochrane handbook. The general methodological quality of each included trials was assessed by six items according to the Cochrane Collaboration's Risk of Bias (ROB) criteria. The items are about randomization, allocation concealment, double blinding, integrity of outcome data, selective reporting, and other bias. Every item is given a possible score of 0 for low, 1 for medium, and 2 for high ROB, all yielding a total score ranging from 0 to 12 for each study. Low ROB is appointed to trials with total score from 0 to 4 , medium ROB with total score from 5 to 8 , and high ROB with total score from 9 to 12 .

2.5. Statistical Analysis. All of the data was calculated by STATA 12.0 software package and Review Manager 5.3 software. The risk ratio (RR) with $95 \%$ CI was applied to analyze the dichotomous data. $\chi^{2}$ and $I^{2}$ tests were used to assess statistical heterogeneity among included studies. If there was no heterogeneity across the trials, the pooled result was obtained by the fixed effect model; otherwise, the random effect model was used. Sensitivity analysis was conducted to estimate the stability of pooled result. Further, we employed Begg's funnel plot and Egger's test to test the publication bias.

\section{Results}

3.1. Search Result. We conducted the systematic research on June 1 , and 251 potentially relevant references were yielded from online database. A total of 90 articles were excluded for duplication and 161 studies were entered next step. After further screening and eligibility assessment, 140 trials were excluded. Finally, 21 trials were selected as appropriate for inclusion in this meta-analysis. The flow chart showing the selection process was presented in Figure 1.

3.2. General Characteristic and Quality Evaluation. The selected trials [13-33] included 2234 advanced NSCLC patients, with 1122 and 1112 in the BJOEI group and control group, respectively, which were all RCTs and conducted in China. Patients' age varied from 18 to 79 years; in addition, the males outnumbered the females. All the included patients were in advanced stage. Nine [13-21] of the studies employed 


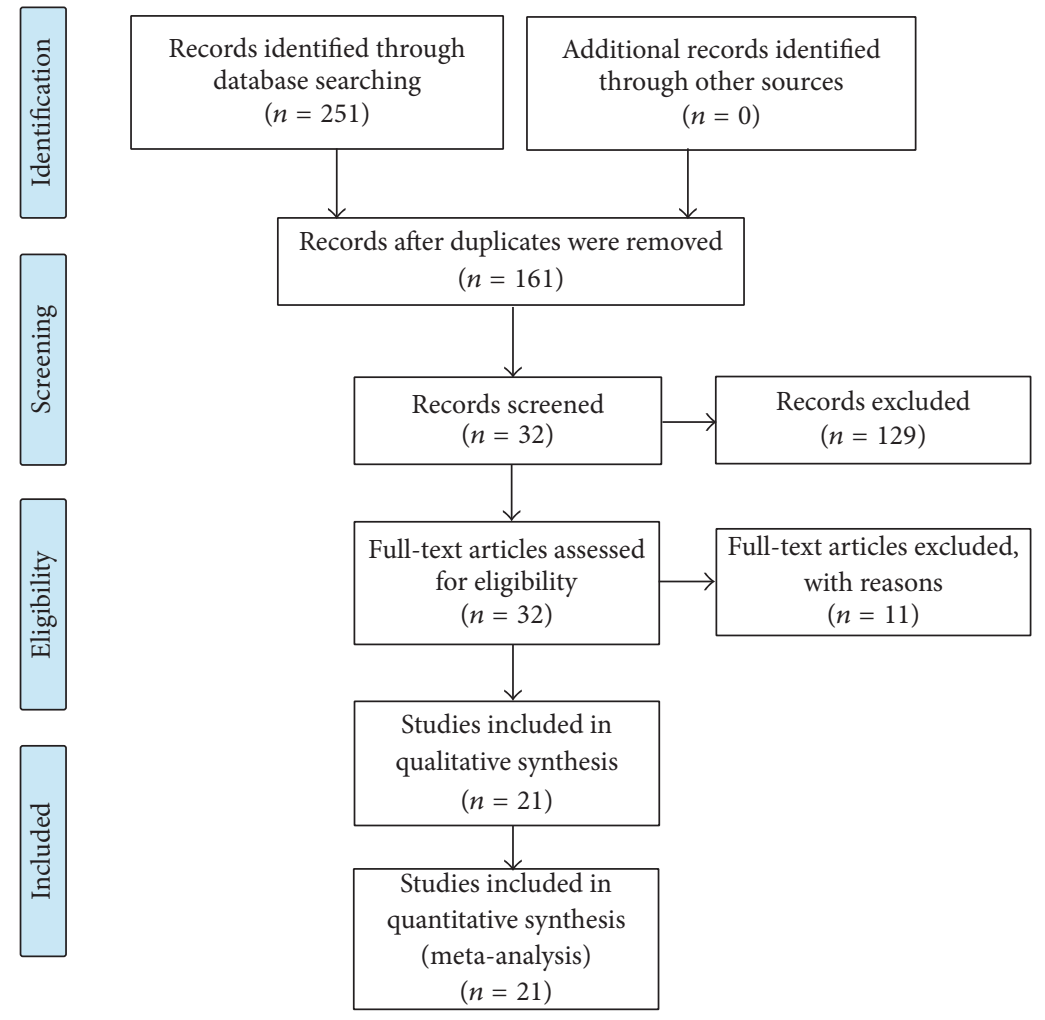

FIGURE 1: Flow chart of searching for included studies.

BJOEI plus GP regimen; four [22-25] employed the BJOEI plus TP regimen; four [26-29] employed BJOEI plus NP regimen; and four [30-33] employed BJOEI plus DP regimen. All of the studies reported the outcome of clinical efficacy; twelve [17-19, 21, 23-26, 29-31, 33] reported the outcome of quality of life; six $[17,19,21-23,25]$ showed the outcome of nausea and vomiting; eight [16, 18, 19, 21-25] provided the outcome of leukopenia; and four [19, 21, 22, 25] provided the outcome of thrombocytopenia. The general characteristics of included trials were shown in Table 1. For quality evaluation, all trials presented moderate ROB. The detailed ROB in different terms of each study was displayed in Table 2.

3.3. Objective Response Rate. All included studies reported ORR in each arm. The heterogeneity analysis showed that no significant heterogeneity was found $\left(I^{2}=0.00 \% ; P=\right.$ $0.98)$, and we applied the fixed effect model in the pooled analysis. As shown in Figure 2, the pooled result indicated a better RR in BJOEI treatment group than in control group $(\mathrm{RR}=1.25 ; 95 \% \mathrm{CI}: 1.14-1.36 ; P<0.00001)$. The results in subgroup analysis of GP regimen $(\mathrm{RR}=1.35 ; 95 \% \mathrm{CI}$ : $1.14-$ $1.59 ; P=0.0004)$ and $\mathrm{DP}$ regimen $(\mathrm{RR}=1.25$; 95\% CI: $1.08-$ $1.45 ; P=0.003$ ) also demonstrated the favorable outcome. However, there were no significant differences with regard to ORR between BJOEI group and control group in subgroup analysis of both TP regimen and NP regimen. The integrated RR for ORR in TP regimen subgroup was 1.17 (95\% CI: 0.89$1.54 ; P=0.26)$ and the pooled $\mathrm{RR}$ for NP regimen group was 1.14 (95\% CI: $0.94-1.37 ; P=0.18$ ). Briefly, this metaanalysis indicated BJOEI plus chemotherapy improved tumor response.

3.4. Improvement of QOL. Twelve studies reported the improvement of KPS. No significant heterogeneity was found among these studies $\left(I^{2}=0.00 \% ; P=0.93\right)$; thus we employed the fixed effect model in this meta-analysis. The pooled result demonstrated that BJOEI combined with chemotherapy could significantly improve the QOL $(\mathrm{RR}=$ 1.87; 95\% CI: $1.63-2.15 ; P<0.00001)$, which was illustrated in Figure 3.

3.5. Grade II or above Nausea and Vomiting. Six studies provided information about nausea and vomiting of the BJOEI treatment group and chemotherapy alone group. The heterogeneity test demonstrated no significant heterogeneity among the studies $\left(I^{2}=0.00 \% ; P=0.67\right)$; thus we used the fixed effect model. As shown in Figure 4, the pooled results indicated that the BJOEI could decrease the risk of developing nausea and vomiting when patients received the chemotherapy combined with BJOEI $(\mathrm{RR}=0.67 ; 95 \% \mathrm{CI}$ : 0.46-0.98; $P=0.04)$.

3.6. Grade II or above Leukopenia. There were eight studies that provided data on Grade II or above leukopenia. The heterogeneity among the included studies was not significant $\left(I^{2}=26 \% ; P=0.22\right)$. As $I^{2}<50 \%$, they were considered 


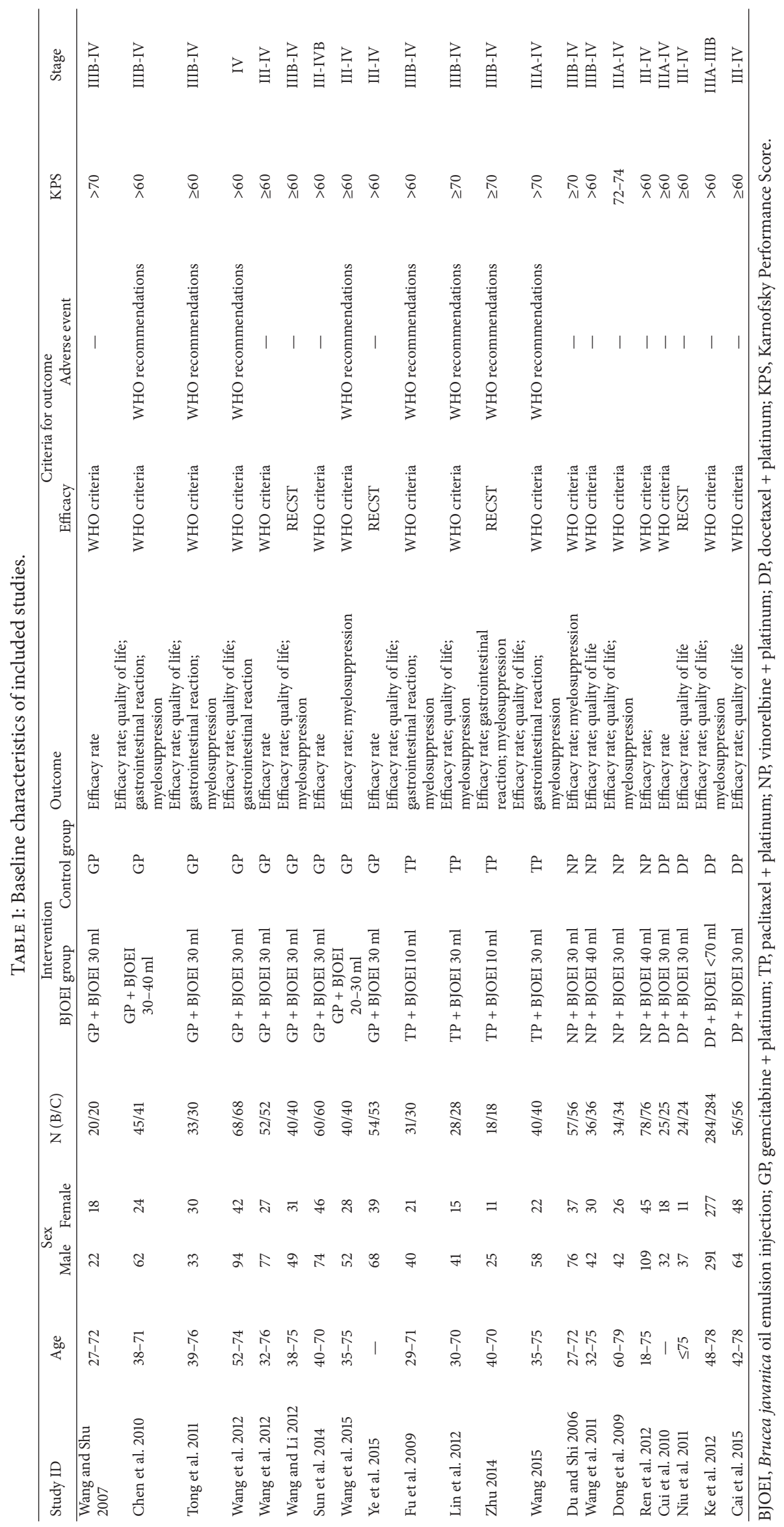


TABLE 2: Risk of Bias scores for included studies.

\begin{tabular}{|c|c|c|c|c|c|c|c|}
\hline Study ID & $\begin{array}{l}\text { Random } \\
\text { sequence } \\
\text { generation }\end{array}$ & $\begin{array}{c}\text { Allocation } \\
\text { concealment }\end{array}$ & $\begin{array}{l}\text { Blinding of } \\
\text { participants } \\
\text { and } \\
\text { personnel }\end{array}$ & $\begin{array}{c}\text { Incomplete } \\
\text { outcome data }\end{array}$ & $\begin{array}{l}\text { Selective } \\
\text { reporting }\end{array}$ & Other bias & Total score \\
\hline \multicolumn{8}{|l|}{ Moderate ROB } \\
\hline Wang and Shu 2007 & 1 & 2 & 2 & 0 & 1 & 1 & 7 \\
\hline Chen et al. 2010 & 1 & 2 & 2 & 0 & 1 & 1 & 7 \\
\hline Tong et al. 2011 & 1 & 2 & 2 & 0 & 1 & 1 & 7 \\
\hline Wang et al. 2012 & 0 & 2 & 2 & 2 & 1 & 1 & 8 \\
\hline Wang et al. 2012 & 0 & 2 & 2 & 0 & 1 & 1 & 6 \\
\hline Wang and Li 2012 & 1 & 2 & 2 & 0 & 1 & 1 & 7 \\
\hline Sun et al. 2014 & 1 & 2 & 2 & 0 & 1 & 1 & 7 \\
\hline Wang et al. 2015 & 0 & 2 & 2 & 0 & 1 & 1 & 6 \\
\hline Ye et al. 2015 & 0 & 2 & 2 & 0 & 1 & 1 & 6 \\
\hline Fu et al. 2009 & 1 & 2 & 2 & 0 & 1 & 1 & 7 \\
\hline Lin et al. 2012 & 2 & 2 & 2 & 0 & 1 & 1 & 8 \\
\hline Zhu 2014 & 1 & 2 & 2 & 0 & 1 & 1 & 7 \\
\hline Wang 2015 & 1 & 2 & 2 & 0 & 1 & 1 & 7 \\
\hline Du and Shi 2006 & 2 & 2 & 2 & 0 & 1 & 1 & 8 \\
\hline Wang et al. 2011 & 1 & 2 & 2 & 0 & 1 & 1 & 7 \\
\hline Dong et al. 2009 & 1 & 2 & 2 & 0 & 1 & 1 & 7 \\
\hline Ren et al. 2012 & 1 & 2 & 2 & 0 & 1 & 1 & 7 \\
\hline Cui et al. 2010 & 1 & 2 & 2 & 0 & 1 & 1 & 7 \\
\hline Niu et al. 2011 & 0 & 2 & 2 & 0 & 1 & 1 & 6 \\
\hline Ke et al. 2012 & 1 & 2 & 2 & 0 & 1 & 1 & 7 \\
\hline Cai et al. 2015 & 2 & 2 & 2 & 0 & 1 & 1 & 8 \\
\hline
\end{tabular}

to be homogeneous and a fixed effect model was employed for analysis (Figure 5). The result showed that the BJOEI combined with the chemotherapy decreased the incidence of Grade II or above leukopenia (RR $=0.63$; 95\% CI: 0.52-0.75; $P<0.00001)$.

3.7. Grade II or above Thrombocytopenia. Four studies provided the data of Grade II or above thrombocytopenia in both arms with no statistical heterogeneity $\left(I^{2}=0 \% ; P=0.91\right)$. Fixed effect model was employed in the meta-analysis. As illustrated in Figure 6, the pooled resulted showed that the BJOEI did not decrease the incidence of Grade II or above thrombocytopenia $(\mathrm{RR}=0.78 ; 95 \% \mathrm{CI}: 0.49-1.23 ; P=0.29$ ).

3.8. Publication Bias. As all the eligible studies reported the outcome of tumor response, we chose to test the potential publication bias among the trials on ORR by Begg's funnel plot and Egger's test. As displayed in Figure 7, the funnel plot was symmetric, suggesting that no evidence of publication bias was found. Moreover, Egger's test provided evidence for no significant publication bias with $P=0.887$.

3.9. Sensitivity Analysis. We performed a sensitivity analysis by sequentially omitting one single study to estimate the summary effect. We conducted the sensitivity analysis on the parameter of ORR for all the studied provided data on it. As shown in Figure 8, the combined effect after exclusion was close to that before exclusion, suggesting that the pooled analysis result was stable.

\section{Discussion}

As a powerful statistical analysis, meta-analysis can yield integrated result from individual study which focuses on the same issue [34]. We performed this meta-analysis to assess the effect of BJOEI plus chemotherapy on tumor response, quality of life, and side effects for advanced NSCLC patients. Twenty-one studies providing data on BJOEI plus chemotherapy versus chemotherapy alone were identified and analyzed comprehensively. As shown above, BJOEI combined with chemotherapy achieved better ORR (RR = 1.25; 95\% CI: 1.14-1.36; $P<0.00001)$ and QOL $(\mathrm{RR}=1.87$; 95\% CI: $1.63-2.15 ; P<0.00001)$, and alleviated Grade II or above toxicity such as nausea and vomiting $(\mathrm{RR}=0.67 ; 95 \%$ CI: $0.46-0.98 ; P=0.04)$ and leukopenia $(\mathrm{RR}=0.63 ; 95 \% \mathrm{CI}$ : $0.52-0.75 ; P<0.00001)$.

Apparently, baselines of the included studies were not consistent for the different chemotherapy regimens that the patients received. Therefore, we carried out a stratified analysis based on chemotherapy regimen for all the included studies on ORR. The results showed that when BJOEI was 


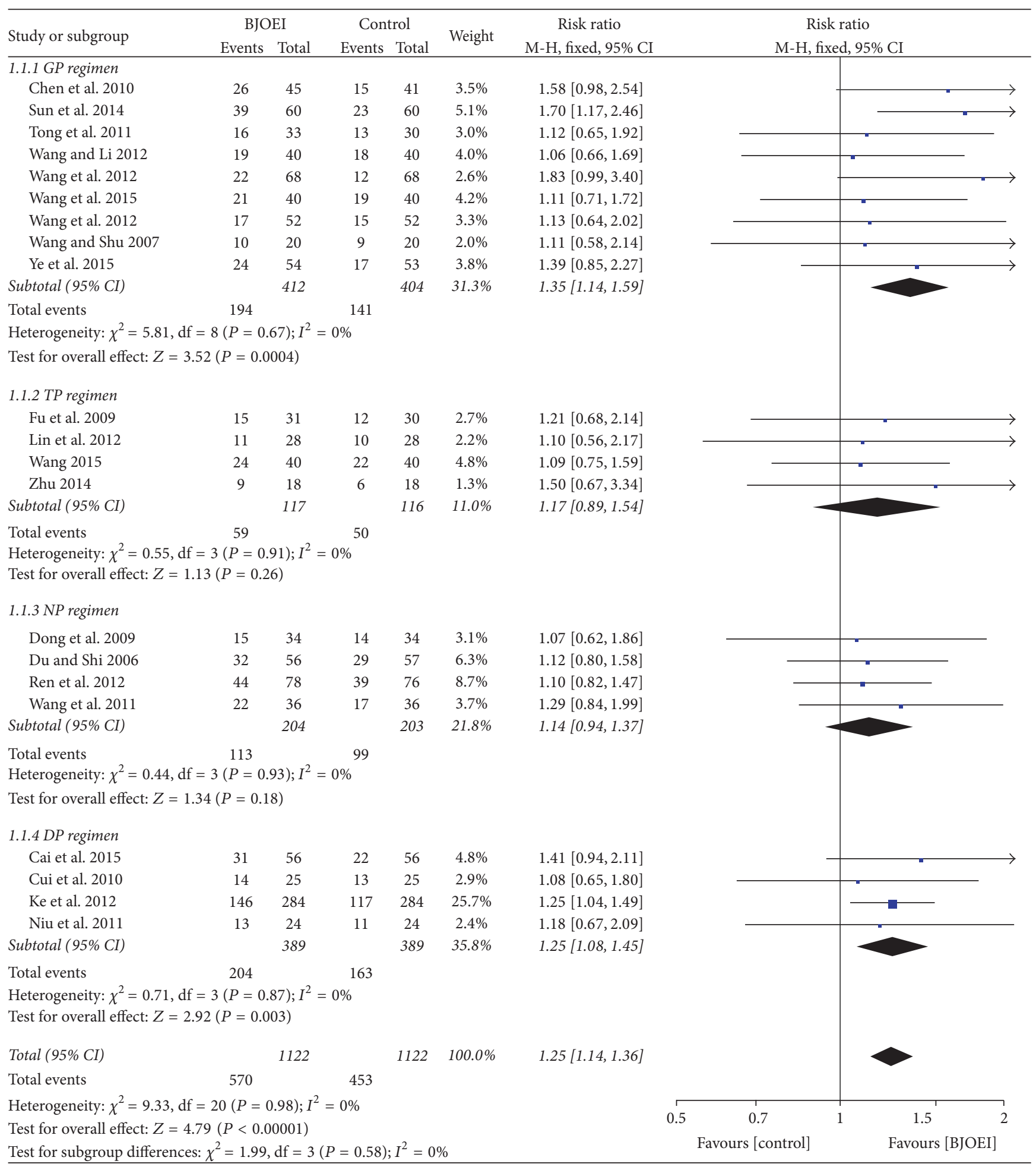

FIgURE 2: Meta-analysis of risk ratio for objective response rate of Brucea javanica oil emulsion injection combined with chemotherapy.

combined with TP regimen and NP regimen, no significant differences $(P=0.26$ and $P=0.18$, resp.) were found between the BJOEI group and control group. However, the pooled result $(P<0.00001)$ and the subgroup analysis of GP regimen $(P=0.0004)$ and $\mathrm{DP}$ regimen $(P=0.003)$ favored the BJOEI combined chemotherapy group. It seems that the sample sizes of TP regimen and NP regimen were too small to test validity. Besides, we evaluated the effect of chemotherapy regimen on quality of life but no significant difference was found, indicating that no matter which regimen the 


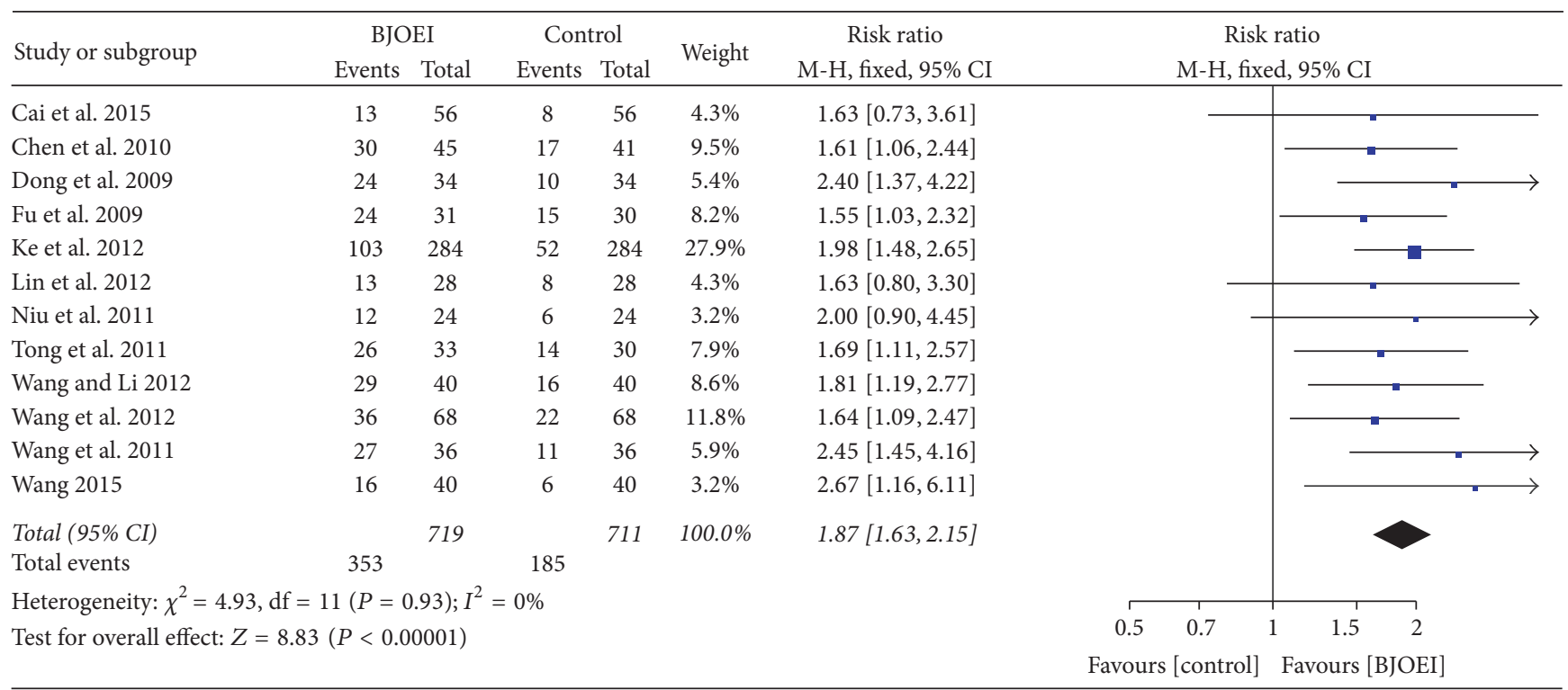

FIGURE 3: Meta-analysis of risk ratio for improvement of quality of life of Brucea javanica oil emulsion injection when combined with chemotherapy.

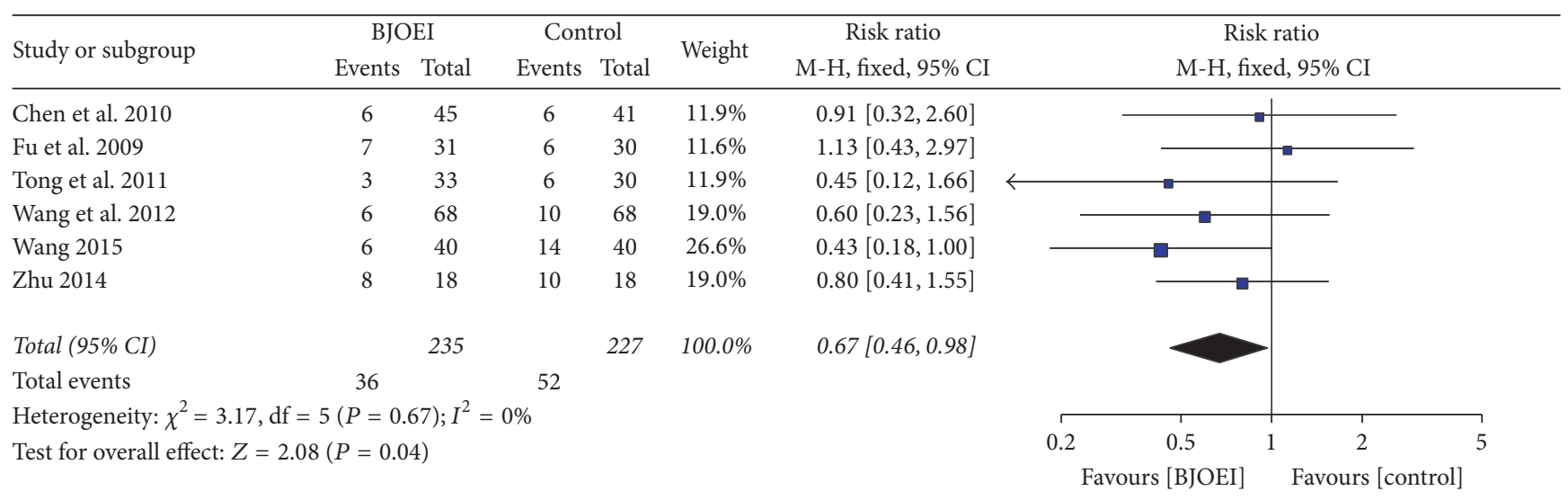

FIGURE 4: Meta-analysis of risk ratio for Grade II or above nausea and vomiting of Brucea javanica oil emulsion injection when combined with chemotherapy.

BJOEI was combined with, the addition of BJOEI during chemotherapy demonstrated a favorable outcome of QOL for advanced NSCLC patients. For publication bias, Begg's funnel plot and Egger's test were also applied in other parameters. The results showed that no publication bias was found for improvement of QOL $(P=0.92)$, nausea and vomiting $(P=0.72)$, leukopenia $(P=0.33)$, and thrombocytopenia $(P=0.94)$.

Brucea javanica oil (BJO) is the main ingredient in BJOEI. In vitro, BJO exhibited a potential ability to kill non-smallcell lung cancer cells [35]. The anticancer activity of BJO might be attributed to the following properties: inducing apoptosis [36], disturbing the cell cycle [36, 37], disrupting the cellular energy metabolism, and depressing the expression of vascular endothelial growth factor. Though the precise mechanism of this anticancer drug is poorly understood, our meta-analysis, together with the previous comprehensive analysis [38], corroborated the efficiency of BJOEI in clinical practice. In Wang's meta-analysis, twenty-two studies fulfilled the inclusion criteria and the pooled results showed that the addition of BJOEI during chemotherapy for NSCLC significantly increased the objective response rate $(\mathrm{RR}=$ 1.31; 95\% CI: $1.18-1.45 ; P<0.00001)$, improved the quality of life $(\mathrm{RR}=1.78 ; 95 \% \mathrm{CI}: 1.51-2.09 ; P<0.00001)$, enhanced the immune function, and decreased the incidence of gastroenteric reaction $(\mathrm{OR}=0.59 ; 95 \% \mathrm{CI}$ : $0.44-0.80$; $P=0.0007)$. Different criteria may lead to slightly different finding between these two meta-analyses, but the patients in BJOEI group did demonstrate superior objective tumor response, higher quality of life, and less side effects.

However, the ROB problem of included TCMs studies should be noticed. The quality assessment result indicated 


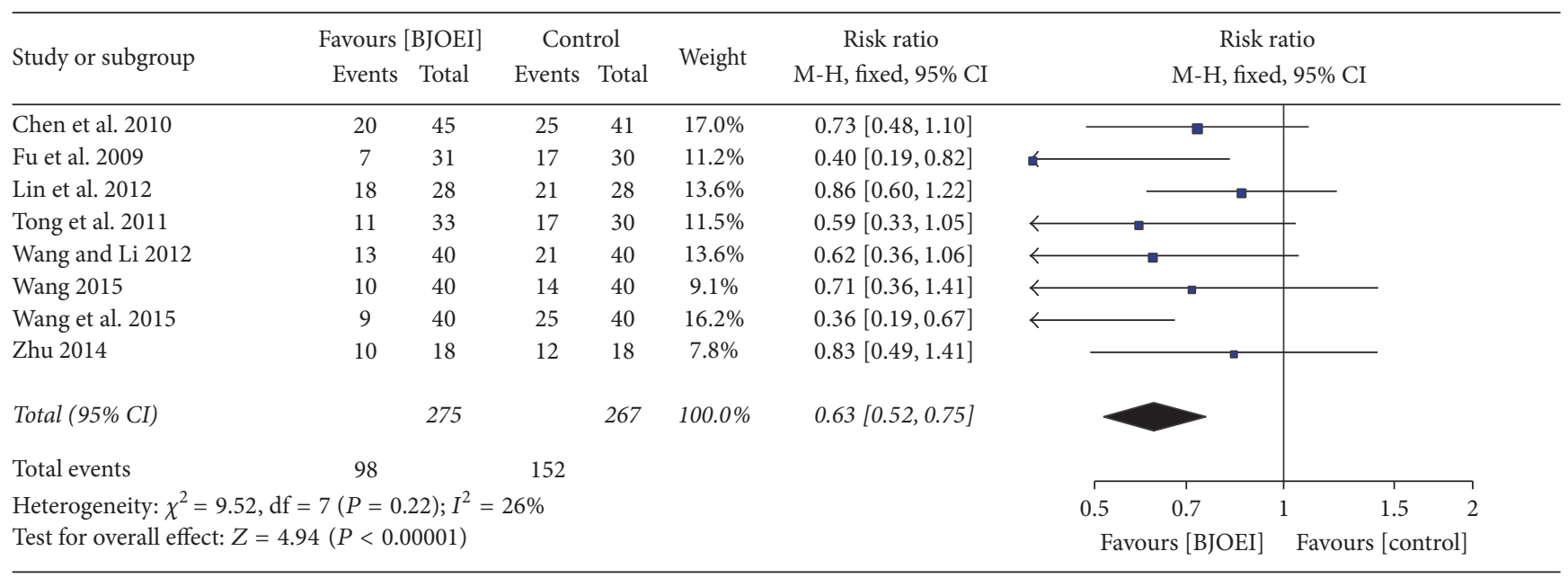

FIgURE 5: Meta-analysis of risk ratio for Grade II or above leukopenia of Brucea javanica oil emulsion injection when combined with chemotherapy.

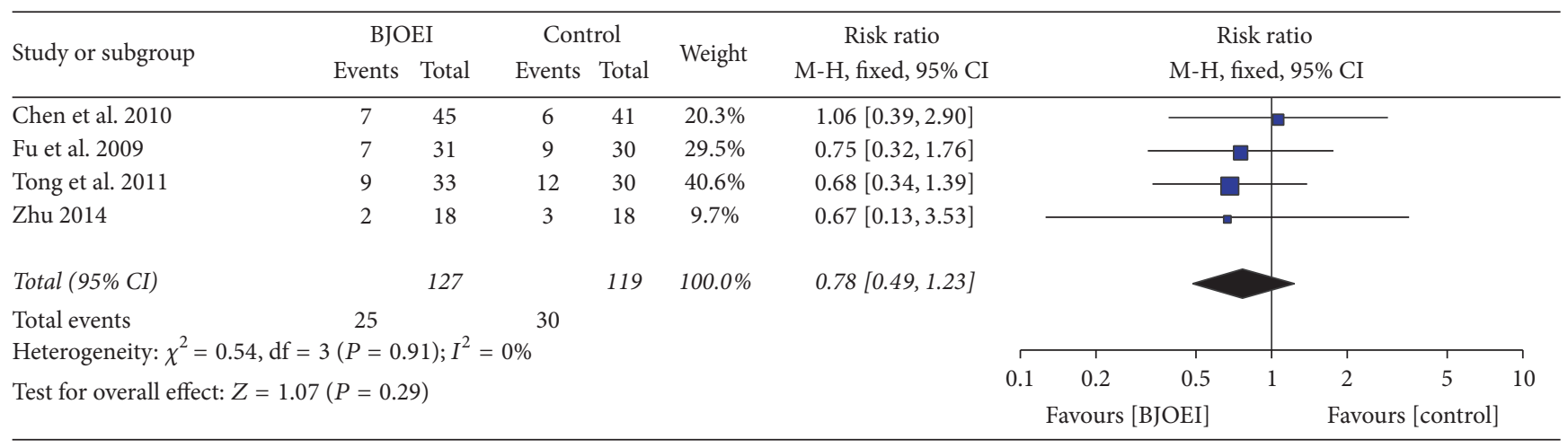

FIGURE 6: Meta-analysis of risk ratio for Grade II or above thrombocytopenia of Brucea javanica oil emulsion injection when combined with chemotherapy.

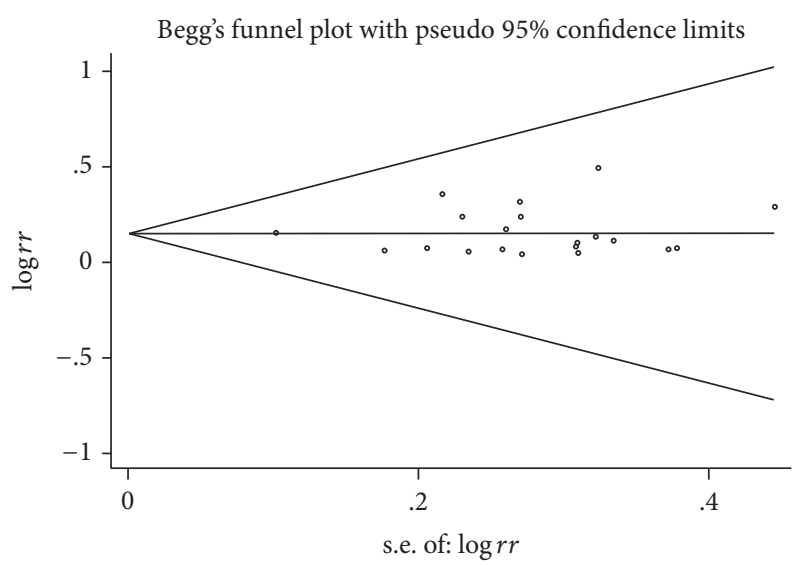

FIgURE 7: Publication bias of the included studies: funnel plot of objective response rate.

that no trial with low ROB was included, but all trails were with moderate ROB. Obviously, the relatively high risk of bias, resulting from faulty design of randomization, loss of double blinding, and allocation concealment, as well as the incertitude of patients' withdrawal, undermined the credibility of the synthesized results of our meta-analysis. McCulloch et al. [39] found that not accounting for ROB would have magnified the evidence of benefit and failed to detect nonsignificance of results. In their research, comparing with those with low ROB, studies with high $\mathrm{ROB}$ overestimated the efficacy of Chinese herbal medicines during fluorouracil-based chemotherapy for colorectal cancer by $16 \%$ improvement in tumor response $(\mathrm{RR}=1.39,95 \% \mathrm{CI}$ : $1.18-1.63$ versus $\mathrm{RR}=1.20$, 95\% CI: $0.81-1.79)$, nearly $2-$ fold reduction of platelet toxicity $(\mathrm{RR}=0.35,95 \% \mathrm{CI}$ : $0.15-$ 0.84 versus $\mathrm{RR}=0.65,95 \% \mathrm{CI}: 0.11-3.92), 2$-fold reduction of vomiting toxicity $(\mathrm{RR}=0.45,95 \% \mathrm{CI}$ : $0.33-0.61$ versus $\mathrm{RR}=0.87,95 \% \mathrm{CI}: 0.48-1.58$ ), and $21 \%$ reduction in diarrhea toxicity $(\mathrm{RR}=0.34,95 \% \mathrm{CI}: 0.20-0.58$ versus $\mathrm{RR}=0.43$, 95\% CI: 0.19-1.01). Although the quality of reporting RCTs of TCMs has been enhanced in the past decade, more and more academics realize that the percentage of high quality reports remains low, exacerbating the ROB problem relevant to 


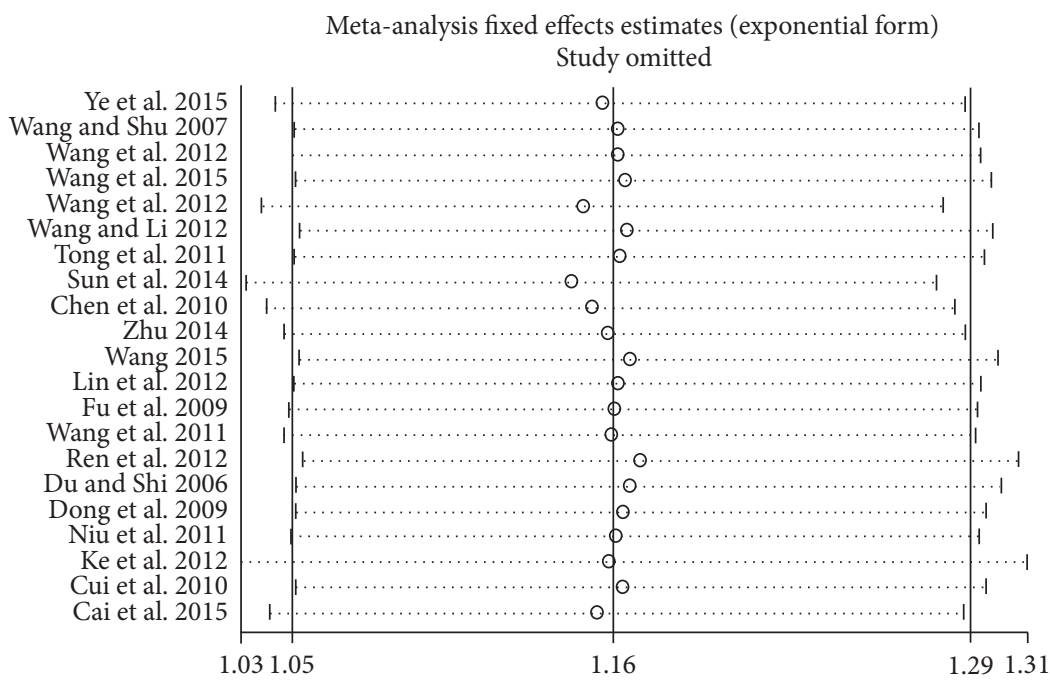

Figure 8: Sensitivity analysis of the included studies.

TCMs trials [39-41]. Researchers should take responsibility for making registration of clinical trials [42], paying more attention to experiment design and methodological quality [43], and receiving education to write high quality report, so as to increase the credibility of TCMs studies.

Actually, limitations exist in our meta-analysis. First, we regarded EMBASE, PubMed, and the conference proceeding of ASCO as main sources of eligible studies, but all the included studies comparing BJOEI plus chemotherapy with chemotherapy alone for advanced NSCLC patients were searched in Chinese academic database. Second, double blinding and allocation concealment were not developed and implemented in all studies, incurring potential risks of selection bias and impairing the quality of this meta-analysis. Third, some of the studies which did not mention detailed characteristics of patients' age and gender distribution might also raise the risk of bias.

\section{Conclusion}

As one of the TCMs, BJOEI has been widely employed in China for many years. In recent year, the clinical practice indicated that the combination of BJOEI and chemotherapy not only improved the ORR and QOL, but also reduced the incidence of adverse events for the advanced NSCLC patients. Our meta-analysis did demonstrate and provide objective evidence to support the efficacy of BJOEI. Given that the quality of included studies is not high and there is a ROB problem in the meta-analysis, the anticancer effect of BJOEI should be further confirmed by higher-quality RCTs.

\section{Competing Interests}

The authors declare that there is no conflict of interests regarding the publication of this paper.

\section{Authors' Contributions}

Wei Xu and Xinchan Jiang contributed to the conception and designed the meta-analysis. Wei Xu and Xinchan Jiang collected the data. Wei Xu and Zhengyuan Xu provided the materials/analysis tools. Wei Xu, Xinchan Jiang, and Tong Ye performed the statistical analysis. Wei Xu, Xinchan Jiang, and Qionghua Shi wrote the original thesis. Wei Xu and Xinchan Jiang were responsible for the quality control of the study.

\section{Acknowledgments}

This work was funded by the National Natural Science Foundation of China, according to General Program no. 71273278 , studies on dynamic financing mechanisms of basic medical insurance system for urban residents in China (http://www.nsfc.gov.cn/publish/portal1/tab131/).

\section{References}

[1] L. A. Torre, R. L. Siegel, and A. Jemal, "Lung cancer and personalized medicine," in Lung Cancer Statistics, pp. 11-19, 2016.

[2] W. Q. Chen, R. S. Zheng, P. D. Baade et al., "Cancer statistics in China, 2015," CA: A Cancer Journal for Clinicians, vol. 66, no. 2, pp. 115-132, 2016.

[3] M. J. Li, Q. He, M. Li et al., "Role of gefitinib in the targeted treatment of non-small-cell lung cancer in Chinese patients," Oncotargets and Therapy, vol. 9, pp. 1291-1302, 2016.

[4] X. Y. Wu, V. C. H. Chung, P. Lu et al., "Chinese herbal medicine for improving quality of life among nonsmall cell lung cancer patients: overview of systematic reviews and network metaanalysis," Medicine, vol. 95, no. 1, Article ID e2410, pp. 1-12, 2016.

[5] X. Wang, Z. Huang, H. Li, and X. Cai, "The short-term observation of Shenqifuzheng injection combined with NP chemotherapy in treating elder patients with advanced nonsmall cell lung cancer," Chinese Journal of Lung Cancer, vol. 10, no. 3, pp. 234-236, 2007. 
[6] X. F. Chen, Y. Q. Liu, O. D. Røe et al., "Gefitinib or erlotinib as maintenance therapy in patients with advanced stage non-small cell lung cancer: a systematic review," PLoS ONE, vol. 8, no. 3, Article ID e59314, p. 404, 2013.

[7] S. Akin, G. Can, A. Aydiner, K. Ozdilli, and Z. Durna, "Quality of life, symptom experience and distress of lung cancer patients undergoing chemotherapy," European Journal of Oncology Nursing, vol. 14, no. 5, pp. 400-409, 2010.

[8] C. Tishelman, L.-M. Petersson, L. F. Degner, and M. A. G. Sprangers, "Symptom prevalence, intensity, and distress in patients with inoperable lung cancer in relation to time of death, Journal of Clinical Oncology, vol. 25, no. 34, pp. 53815389, 2007.

[9] F. Qi, L. Zhao, A. Zhou et al., "The advantages of using traditional Chinese medicine as an adjunctive therapy in the whole course of cancer treatment instead of only terminal stage of cancer," BioScience Trends, vol. 9, no. 1, pp. 16-34, 2015.

[10] Y.-Y. Lu, X.-E. Huang, J. Cao et al., "Phase II study on Javanica oil emulsion injection (Yadanzi ${ }^{\circledR}$ ) combined with chemotherapy in treating patients with advanced lung adenocarcinoma," Asian Pacific Journal of Cancer Prevention, vol. 14, no. 8, pp. 47914794, 2013.

[11] Y.-L. Nie, K.-X. Liu, X.-Y. Mao, Y.-L. Li, J. Li, and M.-M. Zhang, "Effect of injection of brucea javanica oil emulsion plus chemoradiotherapy for lung cancer: a review of clinical evidence," Journal of Evidence-Based Medicine, vol. 5, no. 4, pp. 216-225, 2012.

[12] J. P. Zhou and H. X. Yang, "Meta-analysis on efficacy and safety of brucea javanica oil emulsion injection combined with chemotherapy for patients with advanced gastric carcinoma," China Journal of Chinese Materia Medica, vol. 41, no. 2, pp. 326332, 2016.

[13] H. N. Ye, B. L. Qin, M. Su et al., "Clinic effect of brucea javanica oil emulsion combined with chemotherapy in treating advanced non-small cell lung cancer," Guiding Journal of Traditional Chinese Medicine, vol. 21, no. 7, pp. 35-38, 2015.

[14] Y. X. Wang and Q. J. Shu, "Observation of curative effect of brucea javanica oil emulsion combined with GP chemotherapy on treating advanced non-small cell lung cancer," Zhejiang Journal of Integrated Traditional Chinese \& Western Medicine, vol. 17, no. 9, pp. 535-536, 2007.

[15] X. E. Wang, Q. Zhou, Z. Y. Xu et al., "Clinical study on the treatment of non-small cell lung cancer with brucea fruitwater in oil emulsion combined with GP chemotherapy regimen," Chinese Archives of Traditional Chinese Medicine, vol. 30, no. 10, pp. 2260-2263, 2012.

[16] L. C. Wang, X. L. Men, and S. C. Gao, "Clinical observation on the effect of brucea javanica oil emulsion combined with chemotherapy in the treatment for middle-advanced non-small cell lung cancer," Medical Recapitulate, vol. 21, no. 5, pp. 879-881, 2015.

[17] J. H. Wang, G. P. Li, and S. Gong, "Clinical study of bruceolic oil emulsion combined GP program in treatment of advanced non-small cell lung cancer," Chinese Journal of Postgraduates of Medicine, vol. 35, no. 24, pp. 11-13, 2012.

[18] J. C. Wang and D. H. Li, "Clinical observation on brucea javanica oil emulsion combined with GP regimen in the treatment of advanced non-small cell lung cancer," Clinical Medical Engineering, vol. 19, no. 9, pp. 1497-1499, 2012.

[19] W. N. Tong, A. S. Zhuo, and H. X. Zhao, "Curative effect of brucea javanica oil emulsion combined with GP chemotherapy on treating advanced non-small cell lung cancer," People's Military Surgeon, vol. 54, no. 10, pp. 885-887, 2011.

[20] X. H. Sun, P. Gao, Y. F. He et al., "Clinical research on brucea javanica oil emulsion combined with DP chemotherapy in the treatment of advanced NSCLC," Journal of Chinese Medicine, vol. 29, p. 302, 2014.

[21] H. L. Chen, W. P. Wang, Y. P. Lan et al., “The clinical observation of brucea javanica oil emulsion combined with GP regimen chemotherapy in the treatment of advanced non-small cell lung cancer," Journal of Practical Oncology, vol. 25, no. 5, pp. 584-586, 2010.

[22] Y. Q. Zhu, "The observation of recent therapy of brucea javanica oil emulsion combined with TP chemotherapy regimen in treating advanced non-small cell lung cancer," Henan Traditional Chinese Medicine, vol. 34, pp. 260-261, 2014.

[23] L. Wang, "The recent therapy of brucea javanica oil emulsion combined chemotherapy on treating advanced non-small cell lung cancer," Asia-Pacific Traditional Medicine, vol. 11, no. 15, pp. 121-122, 2015.

[24] M. X. Lin, D. X. Chen, and Q. Q. Yang, "Brucea javaniea oil emulsion combined with paclitaxel and cisplatin in treatment of advanced stage non-small cell lung cancer," China Medicine, vol. 7, no. 4, pp. 397-398, 2012.

[25] X. J. Fu, S. Z. Fu, and G. H. Yang, "Clinical observation of brucea javanica oil emulsion combined chemotherapy on treating nonsmall cell lung cancer," Clinical Journal of Medical Officers, vol. 37, no. 4, pp. 635-637, 2009.

[26] K. Wang, X. Long, W. Q. Yang et al., "The recent therapy of brucea javanica oil emulsion combined with DP chemotherapy in 72 patients with advanced lung cancer," Nei Mongol Journal of Traditional Chinese Medicine, vol. 7, pp. 26-27, 2011.

[27] Y. H. Ren, H. Y. Liu, and W. T. Quan, "Effect of fructus brucea oil emulsion combined with chemotherapy of NP regimen in patients with advanced NSCLC," Progress in Modern Biomedicine, vol. 12, no. 21, pp. 4079-4082, 2012.

[28] M. Du and M. Shi, "Curative effect of chemotherapy combined with brucea javanica oil emulsion on treating advanced nonsmall cell lung cancer," Journal of Basic and Clinical Oncology, vol. 19, no. 2, pp. 151-152, 2006.

[29] X. L. Dong, Y. J. Wang, J. Zhou et al., "Clinical study on the treatment of non-small cell lung cancer with NP chemotherapy regimen and brucea fruitwater in oil emulsion," Journal of Xian Jiaotong University, vol. 30, no. 2, pp. 244-246, 2009.

[30] H. Y. Niu, C. P. Wu, J. T. Jiang et al., "Clinical observation of brucea javanica oil emulsion combined with DP chemotherapy in treating advanced non-small cell lung cancer," Chinese Journal of Postgraduates of Medicine, vol. 34, no. 22, pp. 13-16, 2011.

[31] H. Ke, B. Kong, X. Sun et al., "Observation of efficacy of brucea javanica oil emulsion intratumoral injection combined with chemotherapy in the treatment of locally advanced non-small cell lung cancer," Journal of Basic \& Clinical Oncology, vol. 25, no. 2, pp. 130-133, 2012.

[32] H. Z. Cui, Q. Niu, J. Z. Guan et al., “The clinical observation of the patients with medium-term or advanced non-small cell lung cancer treated with Brucea Javanica oil injection combined with chemotherapy," Sichuan Medical Journal, vol. 31, no. 5, pp. 590-591, 2010.

[33] J. Cai, J. F. Lv, H. Liu et al., "The treatment effect of brucea javanica oil emulsion injection combined with chemotherapy in the treatment of advanced non-small cell lung cancer," Chinese 
Journal of Clinical Rational Drug Use, vol. 8, no. 4B, pp. 52-53, 2015.

[34] H. Anbing and X. Ximing, "The effect of ShenQi FuZheng injection in combination with chemotherapy versus chemotherapy alone on the improvement of efficacy and immune function in patients with advanced non-small cell lung cancer: a metaanalysis," PLoS ONE, vol. 11, no. 3, Article ID e0152270, 2016.

[35] L. Zhao, C. Li, Y. Zhang, Q. Wen, and D. Ren, "Phytochemical and biological activities of an anticancer plant medicine: Brucea javanica," Anti-Cancer Agents in Medicinal Chemistry, vol. 14, no. 3, pp. 440-458, 2014.

[36] F. Wang, Y. Cao, H. Y. Liu et al., "Experimental studies on the apoptosis of HL-60 cells induced by Brucea javanica oil emulsion," China Journal of Chinese Materia Medica, vol. 28, no. 8, pp. 759-762, 2003.

[37] X.-J. Yin, H.-Z. Luan, and C.-L. An, "Inhibitory effect of Brucea javanica oil emulsion against cervical cancer cell line Hela and its mechanism," Chinese Journal of Cancer Biotherapy, vol. 15, no. 4, pp. 393-395, 2008.

[38] Q. Wang, M. C. Wang, X. R. He et al., "Meta-analysis on treatment of non-small cell lung cancer with brucea javanica oil emulsion in combination with platinum-contained first-line chemotherapy," China Journal of Chinese Materia Medica, vol. 37, no. 13, pp. 2022-2029, 2012.

[39] M. McCulloch, H. Ly, M. Broffman et al., "Chinese herbal medicine and fluorouracil-based chemotherapy for colorectal cancer: a quality-adjusted meta-analysis of randomized controlled trials," Integrative Cancer Therapies, vol. 15, no. 3, pp. 285-307, 2016.

[40] G. Wang, B. Mao, Z.-Y. Xiong et al., "The quality of reporting of randomized controlled trials of traditional Chinese medicine: a survey of 13 randomly selected journal from mainland China," Clinical Therapeutics, vol. 29, no. 7, pp. 1456-1467, 2007.

[41] J. N. Li, Z. H. Liu, R. Q. Chen et al., "The quality of reports of randomized clinical trials on traditional Chinese medicine treatments: a systematic review of articles indexed in the China National Knowledge Infrastructure database from 2005 to 2012," BMC Complementary and Alternative Medicine, vol. 14, no. 1, article 362, pp. 1-11, 2014.

[42] T. Wu, Y. Li, G. Liu, J. Li, L. Wang, and L. Du, "Chinese clinical trial registry: mission, responsibility and operation," Journal of Evidence-Based Medicine, vol. 4, no. 3, pp. 165-167, 2011.

[43] J. He, L. Du, G. J. Liu et al., "Quality assessment of reporting of randomization, allocation concealment, and blinding in traditional Chinese medicine RCTs: a review of 3159 RCTs identified from 260 systematic reviews," Trials, vol. 12, no. 1, article 122, pp. 286-298, 2011. 


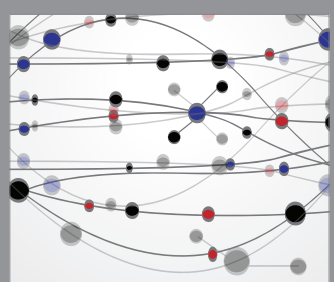

The Scientific World Journal
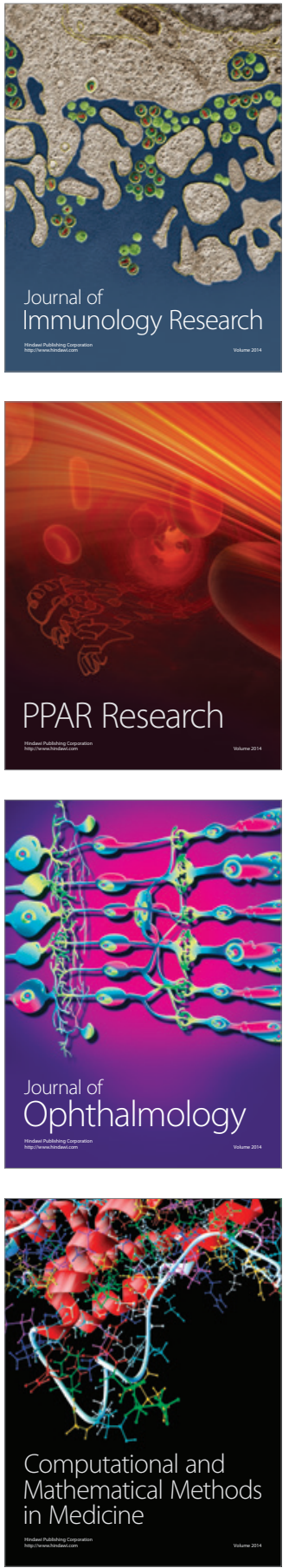

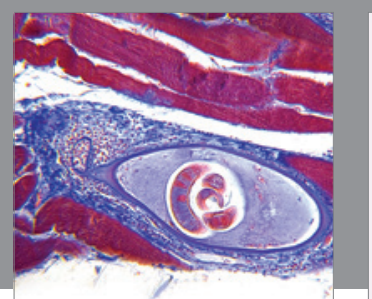

Gastroenterology Research and Practice

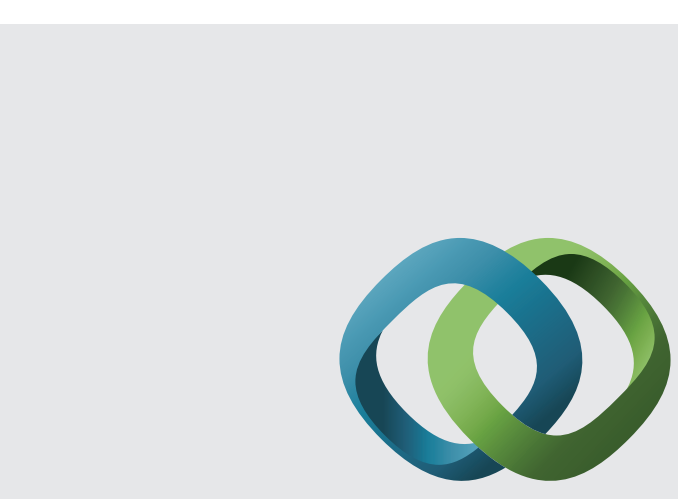

\section{Hindawi}

Submit your manuscripts at

http://www.hindawi.com
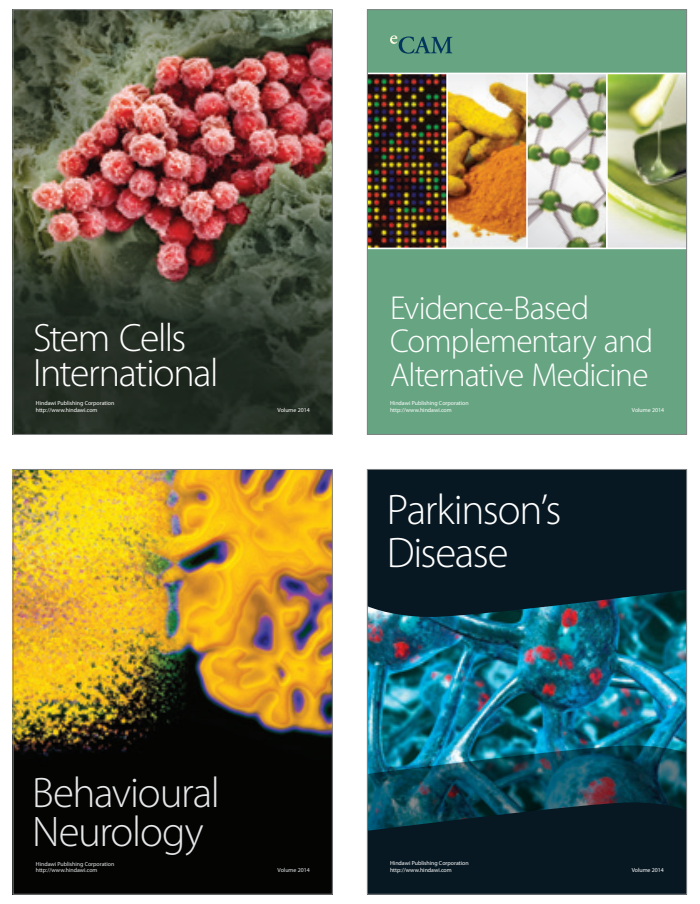
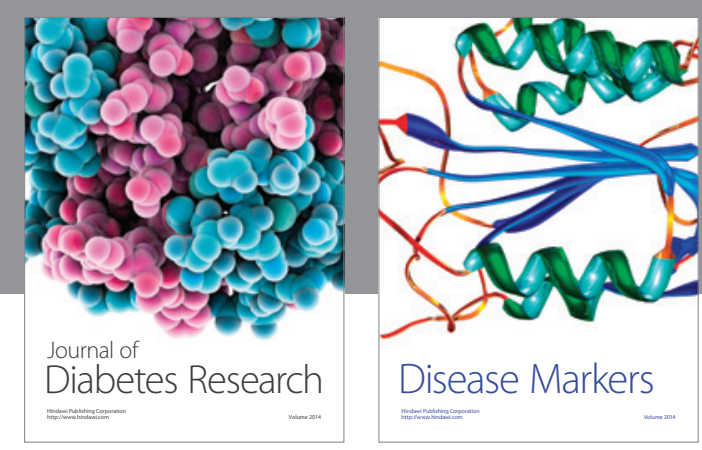

Disease Markers
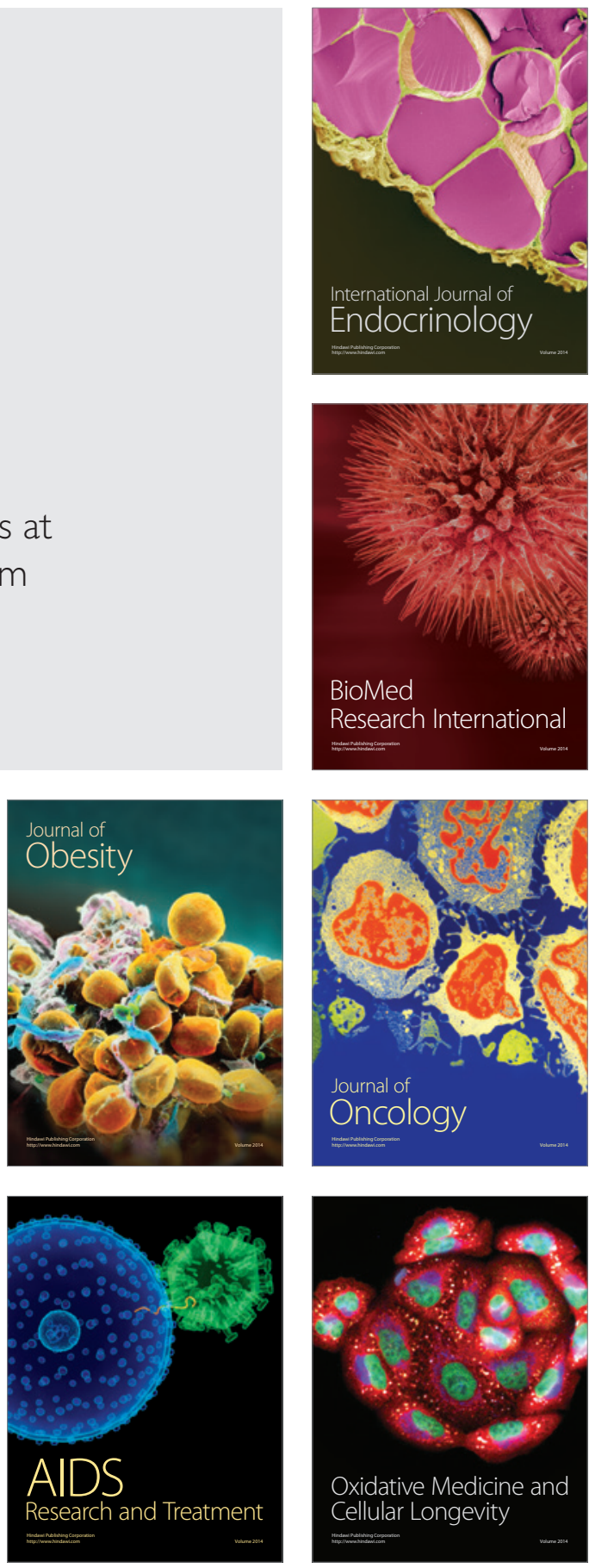